\title{
Reduction of Viral Load in Patients With Acute Sore Throats: Results From an Observational Clinical Trial With Echinacea and Salvia Lozenges
}

Ramon Weishaupt ( $\nabla$ r.weishaupt@avogel.ch )

A.Vogel AG https://orcid.org/0000-0002-7851-5915

Alexandar Buchkov

Diagnostics and Consultation Center Convex

Emil Kolev

Diagnostics and Consultation Center Convex

Peter Klein

DSH-Statistical services $\mathrm{GmbH}$

Roland Schoop

A.Vogel AG

Research

Keywords: Echinacea, Salvia, sore throat, common cold, tonsillopharyngitis, acute therapy, antiviral, viral shedding, respiratory tract infections

Posted Date: April 20th, 2021

DOl: https://doi.org/10.21203/rs.3.rs-425085/v1

License: (9) (1) This work is licensed under a Creative Commons Attribution 4.0 International License. Read Full License 


\section{Abstract}

Background: Acute tonsillopharyngitis or sore throat is an initial and frequent sign of viral respiratory tract infection (RTI) and represents an optimal occasion for early and effective treatment. Most sore throats are caused by a multiplicity of viruses that are highly contagious (e.g., rhinoviruses or coronaviruses). Thus, therapeutic intervention should not only aim to reduce symptoms (antiinflammatory and anesthetic), but also the viral load and transmission (anti-viral). Both of these actions have been attributed to Echinacea purpurea and Salvia officinalis. The goal of this clinical study was to assess the safety and acute treatment effects of the Echinacea/Salvia lozenges for the treatment of early symptoms of sore throat, associated common cold symptoms, and viral shedding from the very beginning.

Methods: A total of 74 patients (age 13-69 years) with acute sore throat symptoms ( $<48 \mathrm{~h}$ ) were treated with five Echinacea/Salvia lozenges per day (4000 mg Echinacea purpurea extract [Echinaforce ${ }^{\circledR}$ ] and $1893 \mathrm{mg}$ Salvia officinalis extract [A. Vogel Switzerland] daily) for 4 days. Patients recorded throat pain, tonsillopharyngitis, and common cold symptoms and provided throat swab samples to assess the viral load before, during and after therapy.

Results: The lozenges had $99 \%$ (very) good ratings and were exceptionally well-tolerated according to the physician and patients. A single lozenge significantly reduced viral load by $62 \%(p<0.03)$, and a 4-day treatment by $96 \%(p<0.02)$. Ninety minutes after the first dose, throat pain was reduced by $48 \%(p<0.001)$ and tonsillopharyngitis symptoms, such as difficulty of swallowing, salivation, and reddening, by $34 \%$ $(p<0.001)$. At the end of treatment, pain, sore throat, and additional common cold symptoms, such as nasal and systemic complaints, were reduced by more than $83 \%(p<0.001)$. The physician's assessment of throat status ultimately confirmed the patient-reported positive treatment outcome. Finally, no complicated RTI developed during therapy, and no antibiotic treatment was required $(0 \%)$.

Conclusions: Echinacea/Salvia lozenges represent an effective option for the early treatment of acute sore throats with a wide safety margin. Importantly, antiviral effects immediately reduce viral shedding and potentially the transmission of RTIs from the very start of treatment.

\section{Background}

Viruses account for the vast majority of respiratory tract infections (RTIs) [1, 2]. A scratchy throat sensation is often described as being the earliest and most frequent indicator of viral infection. This irritation quickly develops into sore throat, (naso-)pharyngitis, or tonsillitis, often accompanied with classical symptoms of the common cold or flu-like illness. A proportion of initial viral complaints exacerbate into bacterial superinfections and associated complications, such as streptococcal $A$ angina [3-6]. Sore throat and associated pathologies are responsible for 200 doctor visits per 1'000 collective annually in the US [7]. The severity of symptoms rapidly increases together with viral shedding, peaking 2-3 days after infection for the common cold [8]. Therapeutic intervention to control symptoms and 
contain viral concentration and subsequent shedding optimally starts with the first signs of infection, i.e. by treating the earliest signs of sore throats.

Non-antibiotic treatment is usually recommended, which mostly relies on anti-inflammatories, anesthetics, or antiseptics that are predominantly administered over-the-counter. They can be administered locally or systemically $[2,9]$. The Echinacea/Salvia lozenge product investigated in this study contains extracts from Echinacea purpurea (Echinaforce $\left.{ }^{\circledR}\right)$ and Salvia officinalis leaves as active ingredients and was flavored with Mentha $\times$ piperita oil.

Echinaforce ${ }^{\circledR}$ is a standardized extract from freshly harvested Echinacea purpurea root and herb (95\%:5\%) in a $65 \%$ alcohol solution. The most prominent application of Echinaforce ${ }^{\circledR}$ is in the prevention and acute therapy of RTIs [10-13]. Although a detailed mechanism of action has not yet been completely elucidated, in vitro and in vivo studies indicate good virucidal activity against commonly known and newly emerging causative agents of RTIs at physiological concentrations [10,14-18]. Furthermore, Echinaforce extract has been shown to interfere with inflammatory cytokine release, which may help ease typical symptoms of a sore throat, common cold, and flu $[16,19]$.

Salvia officinalis extracts are traditionally used to relieve inflammation in the oral cavity [20]. In addition to the anti-inflammatory and local anesthetic properties [21], they also exert antimicrobial activity against various pathogenic strains, including Staphylococcus aureus, C. albicans, S. typhi, or S. enteritidis [22, 23].

"Echinacea/Salvia Lozenges" are a line extension of Echinaforce ${ }^{\circledR}$ and Salvia extract-containing products especially designed to increase patient convenience. The fixed combination of Echinacea and Salvia in the lozenges equals the composition of "A. Vogel Sore Throat Spray", the effectiveness of which has been proven in a clinical study with a chlorhexidine/lidocaine-containing comparator [24].

The goal of this clinical study was to assess the safety and acute effects of the Echinacea/Salvia lozenges for the treatment of early symptoms of sore throat, associated common cold symptoms, and viral shedding.

\section{Methods}

\section{Patients}

Principally healthy patients aged 12-75 years were recruited with acute symptoms of sore throat lasting no longer than 48 hours and with a minimum of 6 score points on the tonsillopharyngitis symptom severity (TSS) index at inclusion. They were included upon receipt of informed consent (in the case of adolescents, signed by their parents and optionally by the adolescent; in the case of children, signed by their parents). The following exclusion criteria applied: age $<12$ years or $>75$ years; pharyngitis or tonsillitis lasting longer than 48 hours before study start (inclusion); intake of analgesics within the last 12 hours prior to inclusion; use of local sore throat medication within the last 4 hours prior to study start (inclusion); symptoms of primary bacterial pharyngitis or a bacterial superinfection; severe medical 
condition, such as tumors, uncontrolled hypertension, heart insufficiency, or immunosuppressive diseases; systemic use of corticosteroids in the last month prior to study inclusion; allergies to substances used in the lozenges; pregnancy or lactation; participation or inclusion in a clinical trial within the last 30 days.

\section{Formulation and dosing}

One Echinacea/Salvia lozenge contains $800.4 \mathrm{mg}$ of hydro-ethanolic extract (65\% v/v) of freshly harvested Echinacea purpurea (95\% aerial parts and $5 \%$ root, Echinaforce ${ }^{\circledR}$ extract) and $378.5 \mathrm{mg}$ of hydro-ethanolic extract (68\% v/v) of freshly harvested Salvia officinalis leaves (drug extraction ratio 1:17) and excipients. The lozenges were placed into dark brown glass bottles with a PE-screw closure and sealed. Each bottle contained 20 individual lozenges, sufficient for 4 days of consecutive treatment. GMPcompliant manufacturing was performed by A. Vogel AG (Roggwil, CH, Switzerland).

Each participant received a single dose of the study product (1 lozenge) under medical supervision. After completion, they received the rest of the study product sufficient for treatment of sore throat symptoms for 4 consecutive days at a daily dose of 5 times 1 lozenge every 3rd hour a day.

\section{Study design}

This study was designed as an observational, prospective, monocentric clinical trial according to ICH-GCP guidelines and reported by adhering to the guidelines postulated by the STROBE statement for observational studies [25]. The main objective was to assess safety and investigate antiviral and acute treatment effects compared to the pre-treatment condition.

Eligible subjects were recruited from the principal investigator's database and the general practitioners (GPs) contacted in Sofia and adjacent regions. The study was carried out at the Diagnostics and Consultation Center Convex EOOD in Sofia Bulgaria, where participants were referred by their GPs. The study included two study center visits: the inclusion visit (day 1 ) and final closeout visit (day $5 \pm 1$ ).

Demographic data were recorded after signing the informed consent form. Patients were to record study parameters, assess concomitant medication/conditions, and provide a blood sample for safety analysis and an oropharyngeal (OP) swab for virus identification and "baseline" measures. Treatment with $5 \times 1$ Echinacea/Salvia lozenges per day started on day 1 by taking a single lozenge under supervision and recording the acute treatment effects. An OP swab sample was taken again 5 minutes after the first dose. Patients continued with treatment and self-documentation of symptoms at home for 4 consecutive days. Patients were then requested to return to the study center on day $5 \pm 1$ for visit 2 . Diary entries, inclusion and exclusion criteria, and concomitant medication/conditions were checked by the study staff, and patients were to provide another OP swab sample and blood sample for safety analysis and to finally assess the study parameters before final closeout.

\section{Virus identification and load reduction}


OP samples were collected using HydraFlock swabs from PURITAN (Maine, USA) and transferred to a vial containing the transport-storage medium OptiSwab (PURITAN, Maine USA). The viral nucleic acids were extracted from $200 \mu \mathrm{L}$ of each specimen using an Allplex ${ }^{\mathrm{TM}}$ Respiratory kit in accordance with the manufacturer's protocols. The collected specimens were screened for the presence of rhinovirus, influenza, parainfluenza, respiratory syncytial virus (RSV), coronavirus, metapneumovirus (MPV), bocavirus, enterovirus, and adenovirus using a real-time reverse transcription PCR (RT-PCR) kit (Seegene, Inc., Seoul, Korea) in accordance with the manufacturer's protocols.

Responders were defined by the presence of a single virus-positive OP sample (baseline and/or 5 minutes after first intake) that became virus-negative during treatment. The semi-quantitative relative log change and percent reduction in viral load 5 minutes after taking a single lozenge and after 4 days of treatment compared to baseline was calculated from the Ct values derived from RT-PCR measurements in accordance to methods described elsewhere $[26,27]$ using Equations (1) and (2):

$$
\log \text { Change }=\log \left(\frac{1}{2^{(\operatorname{Cr}(t)-C t(0))}}\right)
$$

Percent reduction $(\%)=\left(1-10^{\text {Log Change }}\right) * 100$

where $\mathrm{C} t(0)$ equals the $\mathrm{C} t$ value at baseline and $\mathrm{C} t(\mathrm{t})$ equals the $\mathrm{C} t$ value after 5 minutes or after 4 days. Missing Ct $(\mathrm{t})$ values for responders were set to the detection limit of the RT-PCR setup $(\mathrm{Ct}=42)$ in accordance with the method described by Deffernez [26] for calculating the average log reduction. For one non-responder with a missing value at baseline, we estimated the Ct value using regression.

\section{Throat pain, Tonsillopharyngitis Symptom Severity, and common cold symptoms}

Patients marked throat pain intensity on a $10-\mathrm{cm}$ visual analog scale (VAS) ranging from 0 (no pain) to 10 (most severe pain) at baseline, every 15 minutes for 90 minutes after taking the first lozenge, and twice daily (morning/evening) over 4 days of treatment. A patient was considered "throat pain free" if VAS score was $<1 \mathrm{~cm}$ (responder). The Tonsillopharyngitis Symptom Severity (TSS) scores for throat pain, difficulty swallowing, salivation, reddening, and fever were rated by the patients at baseline, after the first 90 minutes, and twice daily (morning/evening) over 4 days of treatment as 3 ("severe"), 2 ("moderate"), 1 ("mild"), or 0 ("absent"). The intensity of common cold symptoms headache, weakness, fever, sneezing, stuffy nose, runny nose, cough, and throat pain were also rated accordingly at baseline and after 4 days of treatment. The VAS pain scores, individual and aggregated total sum TSS scores, and common cold symptom scores were analyzed. We also determined the number of patients reaching a $50 \%$ reduction in total TSS score (responder) per time point and the average time to reach "absence of any complaint" (total TSS < 1). The investigator assessed the patient's throat status (reddening, swelling, and plaque formation on the throat and tonsils, as well as tenderness and enlargement of lymph nodes) at baseline and after 4 days of treatment for confirmation. 


\section{Concomitant treatments/conditions and incidence of adverse events}

Information on concomitant treatments and adverse events (AEs) were retrieved by the study coordinator during routine contacts, by the investigator during interim and close-out visits, or from the assessment of study diaries. All findings and AEs were coded according to the MedDRA (version 17.0GE). Concomitant medication/therapies were coded according to the MedDRA, M. S. S. O. (2013) [28]. At the final visit, returned study medication was counted to assess compliance. Both the investigator and patient rated the effectiveness and tolerability as 1 ("very good"), 2 ("good"), 3 ("moderate"), or 4 ("poor"). Acceptance was determined by asking patients at visit 2 whether they would like to use the study product again.

\section{Sample size calculations and data evaluation}

The anticipated sample size estimation for a significance level of $a=0.05$ showed that a total TSS score with a standard deviation of 2.5 and a delta of 1 score point, and a VAS score with a standard deviation of $2.2 \mathrm{~cm}$ and a delta of $1 \mathrm{~cm}$ required a sample size of 70 patients to achieve a statistical power of at least $96 \%$. Statistical power calculations were carried out in nQuery Advisor, version 7.0.

Quantitative variables were expressed as means and standard deviations. Binary, categorical, and ordinal parameters were summarized by absolute and relative frequencies. RT-PCR results were calculated as the relative reduction compared to baseline and logarithmically transformed for analysis. Quantitative and qualitative parameters were analyzed per time point in comparison to pre-treatment (baseline) values. Kaplan-Meier analysis was performed at the episode level to determine the time to reach a total TSS score $<1$. All statistical tests were two-sided at a $5 \%$ level of significance and interpreted in a descriptiveexploratory manner using the SAS® system (version 9.4).

\section{Results}

This study was conducted from March 2019 to April 2019. Seventy-five patients with acute sore throats were screened for eligibility. One of the screened patients (1.3\%) dropped out (TSS score $<6$ at inclusion). Thus, a total of 74 patients with acute sore throats completed the study (study collective) and were considered for statistical evaluation.

Table 1 provides the demographic characteristics of the patients enrolled in this study. 
Table 1

Baseline demographic characteristics of study population.

\begin{tabular}{|llll|}
\hline & $\begin{array}{l}\text { Females } \\
(\mathbf{N}=36)\end{array}$ & $\begin{array}{l}\text { Males } \\
\mathbf{( N = 3 8 )}\end{array}$ & $\begin{array}{l}\text { Total } \\
\mathbf{( N = 7 4 )}\end{array}$ \\
\hline Age, years & $37.2 \pm 15.3$ & $33.7 \pm 13.4$ & $35.4 \pm 14.4$ \\
\hline$<18$ years & $4(11.1 \%)$ & $1(2.6 \%)$ & $5(6.8 \%)$ \\
\hline Weight, $\mathrm{kg}$ & $67.4 \pm 14.6$ & $82.7 \pm 12.1$ & $75.3 \pm 15.4$ \\
\hline Height, cm & $165.4 \pm 7.8$ & $180.2 \pm 8.3$ & $173.0 \pm 11.0$ \\
\hline BMl, kg/m² & $24.7 \pm 5.7$ & $25.5 \pm 3.6$ & $25.1 \pm 4.7$ \\
\hline Regular smoker & $17(47.2 \%)$ & $16(42.1 \%)$ & $33(44.6 \%)$ \\
\hline Data are provided as mean \pm standard deviation or $\mathrm{n}(\%)$. \\
\hline
\end{tabular}

\section{Clinical safety outcomes}

In $73(98.6 \%)$ cases, the physicians rated the tolerability of the treatment as "very good"; in 1 patient $(1.4 \%)$ the tolerability was rated as "good" (primary parameter). Patients confirmed the physicians' overall positive ratings, with 73 rating the tolerability (very) good (98.6\%) and 1 patient (1.4\%) rating it as "average".

\section{Adverse events}

During a total of 296 accumulated treatment days, 5 AEs were recorded in 4 of the 74 patients $(5.4 \%)$ : increased ESR "Erythrocyte Sedimentation Rate" $(n=1)$ and CRP "C-reactive protein" $(n=1)$ due to infection, and the known tingling sensation of Echinacea $(n=2)$. None of the AEs required stop of treatment, medication or any other countermeasures. Most of the recorded AEs were of mild to moderate intensity. A single case of non-serious and severe pruritus occurred for 30 minutes after first intake but resolved spontaneously and did not relapse during continuation of treatment; therefore, it was considered unrelated. No serious $A E$ and no serious unexpected adverse drug reactions were recorded.

\section{Virus identification and reduction}

RT-PCR analysis identified 20 virus-positive specimens from 18 individual patients within the 5-day observation period (detection rate: $24.3 \%$ ); 2 patients were positive for two individual virus strains. While accounting for any viral species, one patient cleared the initially positive virus detection after a single lozenge (responder), and 5 responders (27.8\%) were recorded after 4 days of treatment.

A total of 16 datasets were evaluable for semi-quantitative calculation of the virus load reduction, since either the baseline sample or at least 2 of 3 samples were virus positive. An average overall reduction of 
viral mRNA copies (increased Ct values), corresponding to a reduction of viral load was observed 5 minutes after taking a single lozenge and after 4 days of treatment compared to baseline (Table 2). With regard to individual virus strains, a reduction after 5 minutes and 4 days of treatment was observed for rhinovirus, adenovirus, and RSV A. For coronavirus strain NL63, a reduction was observed only after 4 days of treatment.

Table 2

Mean differences in $\mathrm{Ct}$ values compared to baseline $(\Delta \mathrm{Ct})$.

\begin{tabular}{|llll|}
\hline & & $\mathbf{5}$ minutes after taking a single dose & After $\mathbf{4}$ days of treatment \\
\hline Viral strain & $\mathrm{N}$ & Mean $\boldsymbol{\Delta C t}(\mathbf{\pm}$ SD) & Mean $\boldsymbol{\Delta C t}(\mathbf{\pm}$ SD) \\
\hline Coronavirus NL63 & 1 & -0.13 & 17.16 \\
\hline Adenovirus & 3 & $2.54(2.20)$ & $3.12(9.05)$ \\
\hline Rhinovirus & 11 & $0.93(1.70)$ & $3.61(5.11)$ \\
\hline $\begin{array}{l}\text { Respiratory syncytial virus } \\
\text { A }\end{array}$ & 1 & 7.50 & 7.50 \\
\hline Overall & 16 & $2.71(3.37)$ & $7.85(6.51)$ \\
\hline
\end{tabular}

A significant average log reduction of mRNA copies was observed by 5 minutes after taking a single dose $(-0.42 \pm 0.69 ;-62 \%, p=0.0296$, t-test $)$ and after 4 days of treatment $(-1.39 \pm 1.92 ;-96 \%, p=0.0111$, $t$-test $)$ relative to baseline when considering all viruses (Fig. 1).

\section{Throat pain}

Figure 2 shows that the self-reported acute throat pain was continuously alleviated over the first 90 minutes after taking a single lozenge. The baseline throat pain intensity was $5.3 \pm 1.3 \mathrm{~cm}$, which was significantly reduced by $5.9 \%(-0.3 \pm 1.2 \mathrm{~cm} ; \mathrm{p}<0.0334$, t-test $) 15$ minutes after taking a single lozenge and by $48 \%(-2.5 \pm 1.8 \mathrm{~cm} ; \mathrm{p}<0.0001$, t-test $)$ after 90 minutes. Although the pain intensity then increased again by the evening of the first treatment day to a mean $3.9 \pm 1.5 \mathrm{~cm}$, a significant $27 \%$ reduction of throat pain $(-1.42 \mathrm{~cm} ; \mathrm{p}<0.0001$, t-test) compared to baseline still manifested. From day 1 onwards, throat pain reduced gradually, by approximately $20 \%$ each day, until the end of therapy. The throat pain intensity after treatment was $0.5 \pm 1.3 \mathrm{~cm}$, representing a reduction of $4.8 \mathrm{~cm}(-91 \%, p<0.001$, t-test $)$ compared to baseline.

Figure 2 (blue bars) also depicts that 19 patients $(25.7 \%)$ were considered throat pain free (VAS $<1 \mathrm{~cm})$ 90 minutes after taking a single lozenge. In line with direct observation of throat pain intensity, the rate of responders decreased to as few as 4 patients $(5.4 \%)$ by the evening of day 1 , before gradually increasing to 62 patients $(83.8 \%)$ by the end of the treatment.

\section{The TSS score}


Figure 3 shows that patients in this study experienced a total TSS score of $7.3 \pm 1.3$ at baseline, which decreased $34 \%(-2.5 \pm 1.9 ; p<0.0001$, t-test) by 90 minutes after taking a single lozenge. The total TSS score plateaued by the evening of day 1 , before continuously decreasing by approximately $23 \%$ each day, reaching a total TSS score of $0.5 \pm 1.4(-93 \%, p<0.0001$, t-test) by the end of therapy. Eighteen patients (24.3\%) experienced a 50\% reduction in symptom intensity by 90 minutes, and 73 patients (98.6\%) by the end of therapy.

Eleven patients (14.9\%) reached complete absence of any complaints (total TSS $<1$ ) after 3 days of treatment, and the majority of 49 patients (66.2\%) reached it after the end of the therapy.

\section{Common cold symptoms and throat status}

All patients experienced throat pain (inclusion criterion), and most reported additional common cold symptoms, such as nasal symptoms (stuffy nose $93.2 \%$, sneezing $90.5 \%$, and runny nose $68.9 \%$ ) and cough $(90.5 \%)$, at inclusion. Systemic complaints were reported less frequently (headache $40.1 \%$, weakness $32.4 \%$, and fever $9.2 \%$ ). After 4 days of treatment, throat pain completely resolved in $88 \%$ of patients, nasal complaints on average in $83 \%$, and systemic complaints on average in $93 \%$, which is highly consistent with the direct pain assessment on the VAS. Cough completely resolved in $60 \%$ of cases.

Assessment of the throat tissue pathology after 4 days of treatment confirmed remission of the throat and tonsil conditions, with $84 \%$ in the vast majority of cases reported at inclusion. On the other hand, remission of lymph node conditions occurred in $39.3 \%$ of cases reported at inclusion.

\section{Effectiveness and acceptance}

In all cases (100\%), physicians rated the treatment effectiveness as (very) good. Patients mostly confirmed these positive ratings with 70 (94.6\%) giving (very) good ratings. Four patients (5.4\%) rated the effectiveness as "average". Seventy-three patients (98.6\%) stated that they would take the Echinacea/Salvia lozenges again to treat acute sore throat symptoms.

Concomitant medication was documented for 3 patients (4.1\%): expectorants, mucolytics, or other nasal preparations. No antibiotics were applied in the frame of this study, and no cold and/or sore throatassociated exacerbations were detected.

\section{Discussion}

Early treatment of respiratory tract infections is essential for controlling symptomatic progression, as well as viral transmission. Sore throat represents an optimal indicator for starting treatment as the earliest and most bothersome initial symptom. There is a great need for effective and safe over-the-counter measures for the treatment of acute sore throat. This study investigated treatment effects of the Echinacea/Salvia lozenges under real-life conditions compared to pre-treatment and external references. 
The demographic data depicted an ordinary sample of patients included in this study, which was representative for the intended use as over-the-counter self-medication.

In the viral analysis, an increase of $1 \mathrm{Ct}$ unit from baseline corresponds to a log reduction of -0.301 and an approximate $50 \%$ reduction of viral mRNA/viral load, whereas an increase of $3.322 \mathrm{Ct}$ units corresponds to a log reduction of -1 and an approximate $90 \%$ reduction of the viral load [26]. In this regard, taking a single lozenge more than halved the viral load within 5 minutes, demonstrating a very fast and effective reduction in the viral load of the OP cavity. The observed antiviral effect corresponds to earlier in vitro and in vivo observations of specificity against enveloped viruses upon direct contact with Echinaforce extract $[10,14-18]$. Development of resistance was not observed in previous experiments, and the activity appeared to be broad.

The $96 \%$ reduction of viral load observed in this study after 4 days of treatment was comparable to a previous study [29] that reported a $90 \%$ reduction after a maximum 4 days of treatment of the common cold with an iota-carrageenan nasal spray. Although limited in the number of samples, the rate of responders turning virus-negative was superior to the previous study $(27.8 \%$ vs. $13 \%$, respectively, at the same time point). Furthermore, viral shedding of, for example, rhinovirus can last for up to 2 weeks in adults [30]. The viral load in throat swabs peaks around the time of RTI symptom onset, inferring that infectiousness peaks at or shortly before symptom onset [31]. Therefore, active anti-viral treatment from the onset of the first symptoms appears to be critical in reducing the duration of viral shedding and, ultimately, the frequency of transmission.

Lindbaek [32] reported that approximately half (51\%) of patients recovered from a sore throat by day 6 of illness in a cohort of 148 placebo- and bovine colostrum-treated patients (young adults) based on a $1 \mathrm{~cm}$ VAS cut-off for recovery of "throat pain". In this regard, the lozenges were highly efficient in alleviating VAS-reported throat pain, halving complaints already after 90 minutes, with 19 patients (26\%) considered completely pain free (VAS pain $<1 \mathrm{~cm}$ ). We then observed an increase in the average pain intensity until the evening of day 1 . We assume that the pain-relieving effect was transient and speculate that the next intake of a lozenge on day 1 may have been too close to the point in time at which the intensity of the symptoms was assessed for the lozenges to develop their immediate analgesic effects quickly enough. Despite this, throat pain reduced continuously from day 2 onwards, reaching residual levels, with a high proportion of patients $(84 \%)$ considered to be throat pain free after 4 days of treatment. Apparently, the lozenges really need to be taken regularly and permanently, especially at the onset of symptoms, in order to maintain an efficient reduction of throat pain.

In line with observed effects on throat pain, the treatment appeared to be effective to resolve other typical sore throat symptoms assessed by the more extended TSS score, including difficulty swallowing, salivation, throat pain, and reddening/fever, and even common cold symptoms, such as headache, weakness, nasal complaints, or cough. The average total TSS score was significantly reduced after 90 minutes, but the observed reduction was weaker than the VAS-reported throat pain by the same time point, which could be a further indication of a specific and fast "analgesic" effect of the treatment at the 
beginning. The $93 \%$ reduction in the average total TSS score after 4 days of treatment, $93 \%$ reduction in systemic complaints (headache, weakness, fever), and 83\% reduction in nasal complaints (stuffy, nose, runny nose, sneezing) were in line with the VAS-reported throat pain.

After 4 days of consecutive treatment, $66 \%$ of patients had an absence of any throat complaint (total TSS $<1$ ). This was comparable to a previous study [33] in which $36 \%$ of patients had persisting sore throat symptoms from day 5 onwards and slightly superior to Lindbaek's study [32], in which $49 \%$ of patients had persisting symptoms by day 6 and a mean duration of 5-6 days.

Almost three-quarters of the included patients (74.3\%) responded ( $\geq 50 \%$ TSS reduction) after 3 days of treatment. This is highly promising, as Schapowal [24] reported that $63.8 \%$ of patients were responders for a spray of identical Echinacea/Salvia formulation, compared to $57.8 \%$ response rate for a chlorhexidine/lidocaine spray after 3 days in a double-blinded, randomized study. This finding may support lozenges as an at least equally effective, but very likely even more effective, galenic form for the studied Echinacea/Saliva sore throat formulation, especially compared to synthetic comparators [9].

The physicians ultimately confirmed the observed treatment effects described above by documenting pronounced remission in up to $90 \%$ of cases after 4 days of treatment. This finding was highly consistent with the $94.6 \%$ (very) good subjective effectiveness ratings by both the physicians and patients, as well as the fact that 73 of the 74 patients $(98.6 \%)$ would take the lozenges again.

\section{Clinical safety}

We found that the Echinacea/Salvia lozenges had an excellent safety profile for the treatment of acute sore throat and common cold symptoms. They received exceptionally positive tolerability ratings from both the physicians (primary parameter) and patients. This matched well with the low rate of AEs and absence of any serious AEs or unexpected adverse drug reactions, exacerbation, or antibiotic treatment.

\section{Limitations of the study}

This study did not employ an internal comparator or reference treatment and was limited in sample size. Therefore, the causal relationship between the observed treatment effects deduced from the comparison to baseline prior to treatment is limited and should be interpreted with caution until further confirmation in a distinctively larger sample following a controlled set-up. Nevertheless, this study demonstrates promising treatment effects.

\section{Conclusion}

In conclusion, the data presented here indicate that the Echinacea/Salvia lozenge product is safe and well tolerated. The treatment of patients suffering from acute sore throat in this observational cohort was associated with a fast and significant reduction in the intensity of typical symptoms within minutes, and over 4 days of treatment compared to the condition pre-treatment. Application of a single lozenge reduced the viral load by almost half and 4 days of treatment by more than $90 \%$, which may contribute to 
reducing viral shedding and transmission. Patients and physicians may consider the "Echinacea/Salvia Lozenges" as a safe and convenient over-the-counter option for the treatment of acute sore throat and common cold symptoms in children and adults.

\section{Abbreviations}

AE: Adverse event, CRP: C-reactive protein, Ct: cycle threshold, ESR: Erythrocyte sedimentation rate, GCP: Good clinical practice, GMP: Good manufacturing practice, GP: General practitioner, ICH: International council for harmonization, MedDRA: Medical dictionary for regulatory activities, MPV: Metapneumovirus, OP: Oropharyngeal, RSV: Respiratory syncytial virus, RTI: respiratory tract infection, RT-PCR: Real-time reverse transcription polymerase chain reaction, STROBE: Strengthening the reporting of observational studies in epidemiology, TSS: Tonsillopharyngitis symptom severity, VAS: visual analog scale

\section{Declarations}

\section{Ethics approval and consent to participate}

The Local Ethics Committee at Diagnostics and Consultation Center Convex authorized the study protocol (version 1.0, 12 December 2018). No amendments to the study protocol were generated that required additional ethics approval. The clinical study is registered at Clinicaltrials.gov (identifier: NCT04139681).

Written informed consent was obtained from all parents/children, adolescents, and adults before they participated in the study. Accordance with the ethical principles of the Declaration of Helsinki/Good Clinical Practice guidelines, adherence to national/regional regulatory requirements, and data protection were ensured permanently.

\section{Consent for publication}

"Not applicable"

\section{Availability of data and materials}

The datasets used and/or analyzed during the current study are available from the corresponding author on reasonable request.

\section{Competing interests}

R.W. and A.S. are employees of A. Vogel AG, the study sponsor. A.B., E.K., and P.K. received honorarium funds from the study sponsor. The authors indicated that they have no other competing interests to 
declare regarding the content of this article.

\section{Funding}

This study was sponsored by A. Vogel AG, Roggwil, Switzerland. The sponsor covered the funding of the article publication fees. The role of the funder was to supply the study medication, which was the Echinacea/Salvia Lozenges.

\section{Authors' contributions}

A.B. and E.K. collected clinical data and contributed to data analysis. P.K. conducted the statistical analysis and contributed to data analysis. R.W. and R.S. conducted data analysis and wrote the manuscript. All authors contributed to data interpretation and discussion, and read and approved the final manuscript for submission.

\section{Acknowledgements}

The authors thank Dr. Andy Suter and Dr. Dimitar Mirchev for scientific input in the discussion of the results.

\section{References}

1. Jain N, Lodha R, Kabra SK. Upper Respiratory Tract Infections. Indian J Pediatr 2001;68 (12): 11351138.

2. Kuehn BM. IDSA: Avoid Antibiotics for Most Throat Infections. JAMA. 2012;308(13):1307. DOI:10.1001/jama.2012.13019

3. Eccles R. Understanding the symptoms of the common cold and influenza. Lancet Infect Dis. 2005 Nov; 5(11): 718-725.

4. Mäkelä MJ, Puhakka T, Ruuskanen O, Leinonen M, Saikku P, Kimpimäki M, et al. Viruses and Bacteria in the Etiology of the Common Cold. J Clin Microbiol. 1998 Feb; 36(2): 539-542.

5. Georgitis JW. Nasopharyngitis, pharyngitis, and tondillitis. Immunology and Allergy Clinics of North America. 1993;13, 109-118.

6. Bisno AL. Acute Pharyngitis. N Engl J Med 2001; 344:205-211.

7. Armstrong GL, Pinner RW. Outpatient Visits for Infectious Diseases in the United States, 1980 Through 1996. Arch Intern Med. 1999;159(21):2531-2536. DOI:10.1001/archinte.159.21.2531.

8. Heikkinen T, Järvinen A. The common cold. The Lancelet 2003; DOI: https://doi.org/10.1016/S01406736(03)12162-9. 
9. Thomas_M, Del Mar C, Glasziou P. How effective are treatments other than antibiotics for acute sore throat? Br J Gen Pract. 2000 Oct; 50(459): 817-820.

10. Jawad M, Schoop R, Suter A, Klein P, Eccles R. Safety and Efficacy Profile of Echinacea pur-purea to Prevent Common Cold Episodes: A Randomized, Double-Blind, Placebo-Controlled Trial. EvidenceBased Complementary and Alternative Medicine. Volume 2012, Article ID 841315, 7 pages. doi:10.1155/2012/841315.

11. Raus K, Pleschka S, Klein P, Schoop R, Fisher P. (2015). Effect of an Echinacea-Based Hot Drink Versus Oseltamivir in Influenza Treatment: A Randomized, Double-Blind, Double-Dummy, Multicenter, Noninferiority Clinical Trial. Curr Ther Res Clin Exp. 2015 Apr 20;77:66-72.

12. Schapowal A. Efficacy and safety of Echinaforce ${ }^{\circledR}$ in respiratory tract infections. Wien Med Wochenschr (2013) 163:102-105.

13. Schapowal A, Klein P, Johnston S.L. (2015). Echinacea Reduces the Risk of Recurrent Respiratory Tract Infections and Complications: A Meta-Analysis of Randomized Controlled Trials. Advances in Therapy 32(3), 187-200.

14. Signer J, Jonsdottir HR, Albrich WC, Strasser M, Züst R, Ryter S, et al. In vitro virucidal activity of Echinaforce ${ }^{\circledR}$, an Echinacea purpurea preparation, against coronaviruses, including common cold coronavirus 229E and SARS-CoV-2. Virol J. 17, 136 (2020).

15. Pleschka S, Stein M, Schoop R, Hudson JB. Anti-viral properties and mode of action of standardized Echinacea purpurea extract against highly pathogenic avian influenza virus (H5N1, H7N7) and swine-origin H1N1 (S-OIV). Virol J. 2009;6:197.

16. Sharma M, Anderson SA, Schoop R, Hudson JB. Induction of multiple pro-inflammatory cytokines by respiratory viruses and reversal by standardized Echinacea, a potent antiviral herbal extract. Antiviral Res. 2009 Aug;83(2):165-70.

17. Sharma SM, Anderson M, Schoop R, Hudson JB. Bactericidal and anti-inflammatory proper-ties of a standardized Echinacea extract (Echinaforce $\left.{ }^{\circledR}\right)$ : Dual actions against respiratory bacteria. Phytomedicine. 2010 Jul;17(8-9):563-8. DOI: 10.1016/j.phymed.2009.10.022. Epub 2009 Dec 29.

18. Ogal M, Johnston SL, Klein P, Schoop R. Echinacea Reduces Antibiotic Usage in Children through Respiratory Tract Infection Prevention: A Randomized, Blinded, Controlled Clinical Trial. Under review by; European Journal of Respiratory Medicine. 2018.

19. Sharma, M., Schoop, R. and Hudson, J.B. (2009b). The efficacy of Echinacea in a 3-D tissue model of human airway epithelium. 26 May 2010. https://doi.org/10.1002/ptr.3051.

20. European Union herbal monograph on Salvia officinalis L., folium. 2 February 2016; EMA/HMPC/277152/2015. Draft - Revision.

21. Miraj S, Kiani S. A review study of therapeutic effects of Salvia officinalis L. Der Pharmacia Lettre, 2016, 8 (6):299-303.

22. Delamare AP, Moschen-Pistorello IT, Artico L, Atti-Serafini L, Echeverrigaray S. Antibacterial activity of the essential oils of Salvia officinalis L. and Salvia triloba L. cultivated in South Brazil. Food Chemistry. Volume 100, Issue 2, 2007;Pages 603-608. 
23. Bozin B, Mimica-Dukic N, Samojlik S, Jovin E. Antimicrobial and Antioxidant Properties of Rosemary and Sage (Rosmarinus officinalis L. and Salvia officinalis L., Lamiaceae) Essential Oils. J. Agric. Food Chem. 2007;55, 19, 7879-7885.

24. Schapowal A, Berger D, Klein P, Suter A. Echinacea/sage or chlorhexidine/lidocaine for treating acute sore throats: a randomized double-blind trial. Eur J Med Res. 2009 Sep 1;14(9):406-12.

25. Von Elm E, Altman DG, Egger M, Pocock SJ, Gøtzsche PC, Vandenbroucke JP. The Strengthening the Reporting of Observational Studies in Epidemiology (STROBE) statement: guidelines for reporting observational studies. Lancet 2007; 370: 1453-57

26. Deffernez C, Wunderli W, Thomas Y, Yerly S, Perrin L, Kaiser L. Amplicon Sequencing and Improved Detection of Human Rhinovirus in Respiratory Samples. J Clin Microbiol. 2004 Jul; 42(7): 32123218.

27. Ludwig M, Enzenhofer E, Schneider S, Rauch M, Bodenteich A, Neumann K, Prieschl-Grassauer E, Grassauer A, Lion T, Mueller CA. Efficacy of a Carrageenan nasal spray in patients with common cold: a randomized controlled trial. Respir Res. 2013 Nov 13;14(1):124.

28. MedDRA, M. S. S. O. (2013). Introductory Guide for Standardised MedDRA Queries (SMQs) Version 16.1 Chantilly, VA: MedDRA Maintenance and Support Services Organization.

29. Eccles R, Winther B, Johnston SL, Robinson P, Trampisch M, Koelsch S. Efficacy and safety of iotacarrageenan nasal spray versus placebo in early treatment of the common cold in adults: the ICICC trial. Respir Res. 2015 Oct; 16(1): 1-11.

30. Lu X, Holloway B, Dare RK, Kuypers J, Yagi S, Williams JV, Hall CB, Erdman DD. Real-Time Reverse Transcription-PCR Assay for Comprehensive Detection of Human Rhinoviruses. J Clin Microbiol. 2008 Feb; 46(2): 533-539.

31. He X, Lau E, Wu P, Deng X, Wang J, Hao X. Temporal dynamics in viral shedding and transmissibility of COVID-19. Nat Med. 26, 672-675 (2020).

32. Lindbaek M, Francis N, Cannings-John R, Butler CC, Hjortdahl P. Clinical course of suspected viral sore throat in young adults: Cohort study, Scandinavian Journal of Primary Health Care, 24:2, 93-97, DOI: 10.1080/02813430600638227.

33. Little P, Gould C, Williamson I, Warner G, Gantley M, Kinmonth AL. Clinical and psychosocial predictors of illness duration from randomised controlled trial of prescribing strategies for sore throat. BMJ 1999;319(7212):736-737.

\section{Figures}




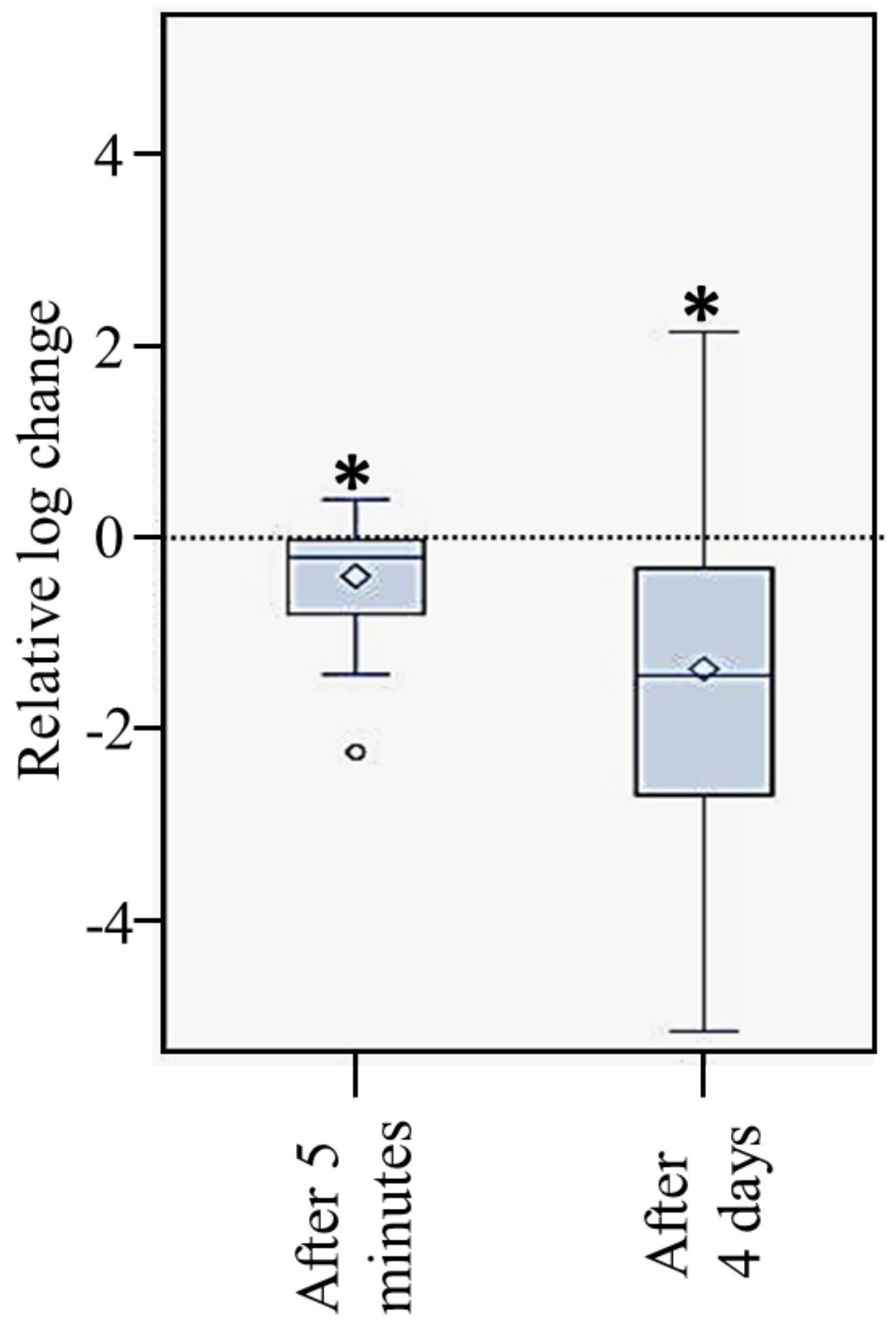

Figure 1

Relative log change in viral load compared to baseline. The boxplots convey median \pm minimal and maximal values, rhombus icons represent means, ${ }^{*} p<0.05$. 


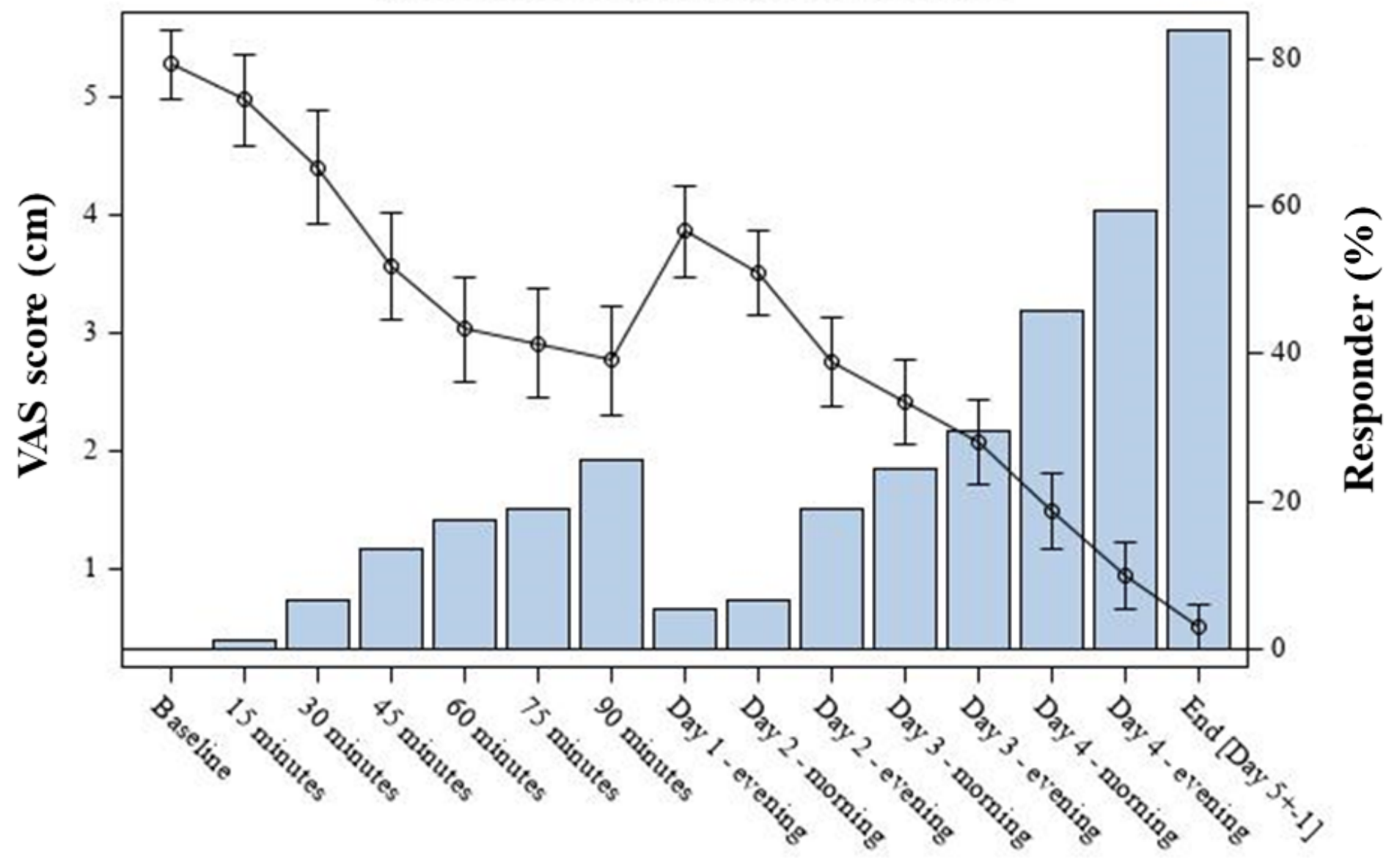

Figure 2

Frequency of treatment responders (blue bars) and mean VAS scores over time (solid line). Responders were defined as VAS score $<1 \mathrm{~cm}$. Mean VAS scores are shown with $95 \%$ confidence intervals. 


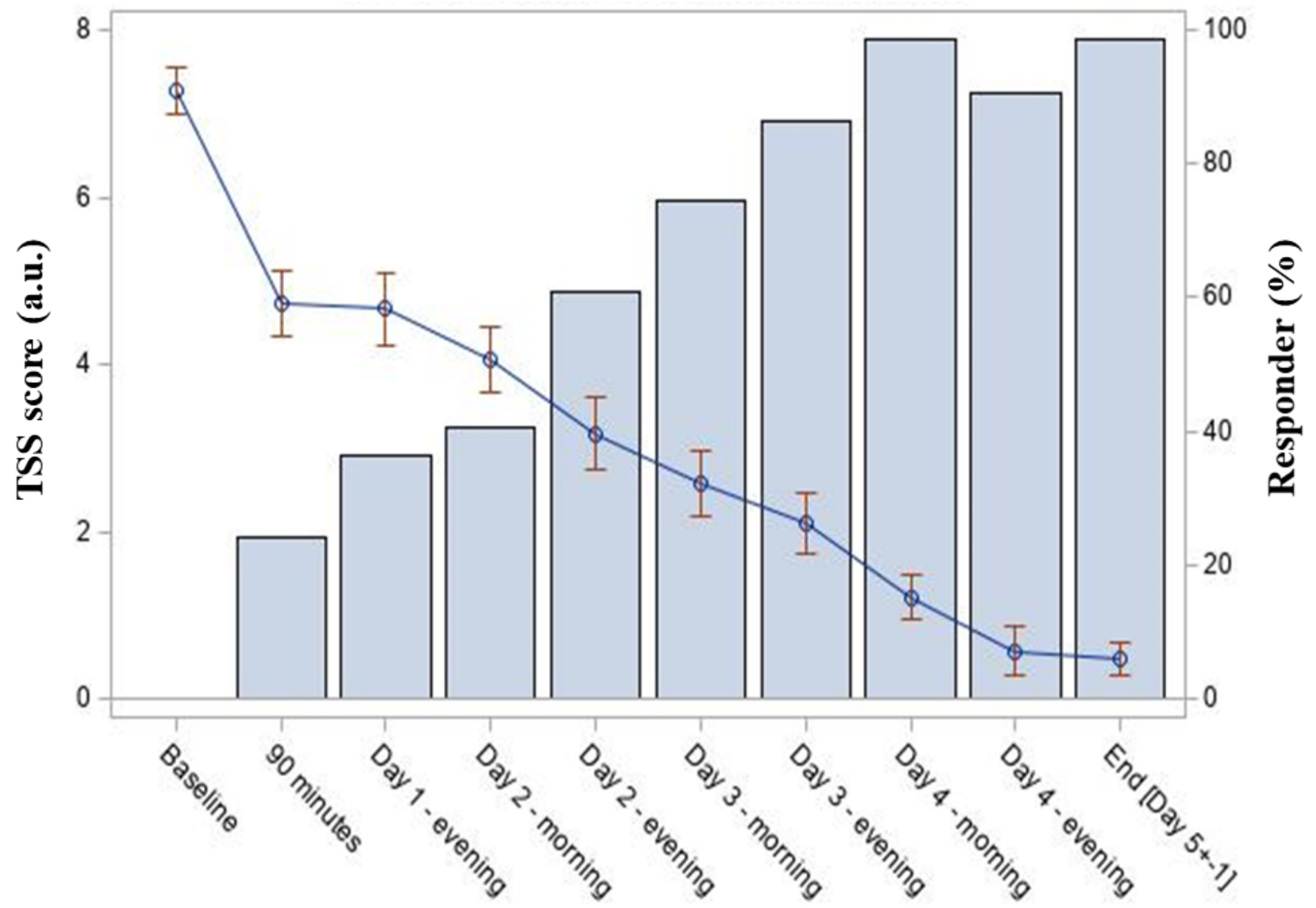

Figure 3

Frequency of patients reaching $\geq 50 \%$ total TSS reduction (blue bars) and mean total TSS score (solid line) over time. Mean TSS score is shown with $95 \%$ confidence intervals. 